\title{
Leigh syndrome: A rare mitochondrial disorder
}

\author{
Gopen Kumar Kundu, Amina Akhter, Shaheen Akhter and Md. Mizanur Rhaman
}

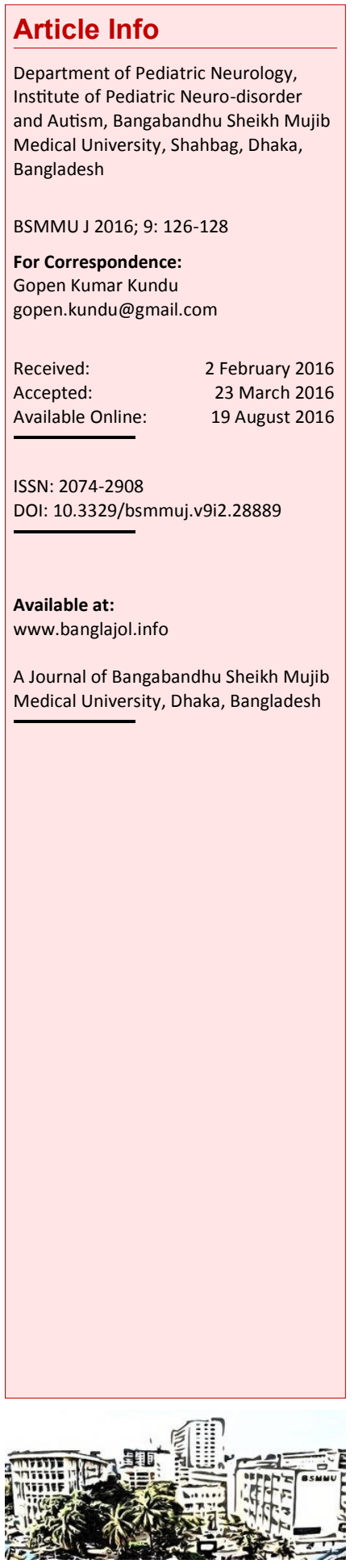

\section{Abstract}

A case of Leigh syndrome, mitochondrial disorder of childhood, was reported with regression of motor development and involuntary movements characterized by slow writhing movement of both upper and lower limbs. There was slightly raised ammonia level $(64.0 \mu \mathrm{mol} / \mathrm{L})$. Serum lactate was increased $(5.2 \mathrm{mmol} / \mathrm{L})$. Magnetic resonance imaging showed hyperintense lesion in the basal ganglia, midbrain and dentate nuclei of cerebellum that lead to the diagnosis of Leigh syndrome.

\section{Introduction}

The Leigh disease is a rare mitochondrial disorder termed as subacute necrotizing encephalitis. It was first described by Denis Leigh in 1951.1 The clinical presentation of Leigh syndrome is manifested by psychomotor delay/regression, strabismus, muscular hypotonia, nystagmus, swallowing difficulty, ataxia, respiratory insufficiency, pyramidal signs, acute deterioration following common infections and lactate acidemia. In Leigh disease, the respiratory chain enzymes defect is responsible. These are due to deficiency of cytochrome-coxidase, pyruvate dehydrogenase and biotinidase. 2

The patterns of inheritance are autosomal recessive, $\mathrm{X}$-linked recessive and mitochondria. 3 The neuropathological features of Leigh disease are identical. These are necrotic lesions (bilateral, focal and symmetric) with demyelination, vascular proliferation and gliosis in the basal ganglia, diencephalon and cerebellum and brainstem. $\underline{4}$ The diagnosis is usually done based on clinical features, meta-bolic abnormalities, mode of inheritance and neuroimaging findings. $\frac{5}{-}$

\section{Case Report}

\section{Presentation}

A 26-month-old immunized boy of consanguineous mating parents from a rural area of Bangladesh admitted into the Bangabandhu Sheikh Mujib Medical University with the complaints of regression of motor development (loss of neck control, unable to sit and stand which was previously achieved) and involuntary movements characterized by slow writhing movement of both upper and lower limbs for 1 month.
He had a history of repeated vomiting and respiratory distress since 7 month of his age. For this, he was admitted several times in specialized hospital but no significant improvement was observed. It was diagnosed as a case of neurodegenerative disease. He had no history of convulsion or trauma to the head. His perinatal period was uneventful and the growth was normal up to 7 months of his age His mother stated that she lost her 3 sibs $(2,4$ and 2 years of their age) due to gastrointestinal tract or respiratory complains.

Examination findings revealed that he was conscious and co-operative. Involuntary movement (choreoathetosis) of hand was present in both hands and legs. Vitals signs were within normal limit. Hair was brownish in color. Eye-ENT findings were normal. BCG mark was present. On examination of the nervous system, he was babinski signs were positive. Developmental assessment revealed normal gross motor and fine motor activities. The child started speech at the age of 12 month. Vision and hearing were intact.

\section{Investigations}

Investigational reports showed slightly raised ammonia level $(64.0 \mu \mathrm{mol} / \mathrm{L})$. Serum lactate was increased $(5.2 \mathrm{mmol} / \mathrm{L})$. Blood glucose level was normal. Urinary ketones were absent. CPK level was within normal limit. MR imaging of brain (T2 and flair image) revealed hyperintence lesion in basal ganglia, midbrain, medulla and dentate nuclei of cerebellum indicating mitochondrial encephalopathy (Figure 1). MR spectoscopy showed high lactate peak suggestive of subacute necrotizing encephalomyelopathy (Leigh disease; Figure 2).

IMD panel (TMS) with biotinidase screening were normal. Urinary organic acid analysis hypotonic but jerks were exaggerated and 


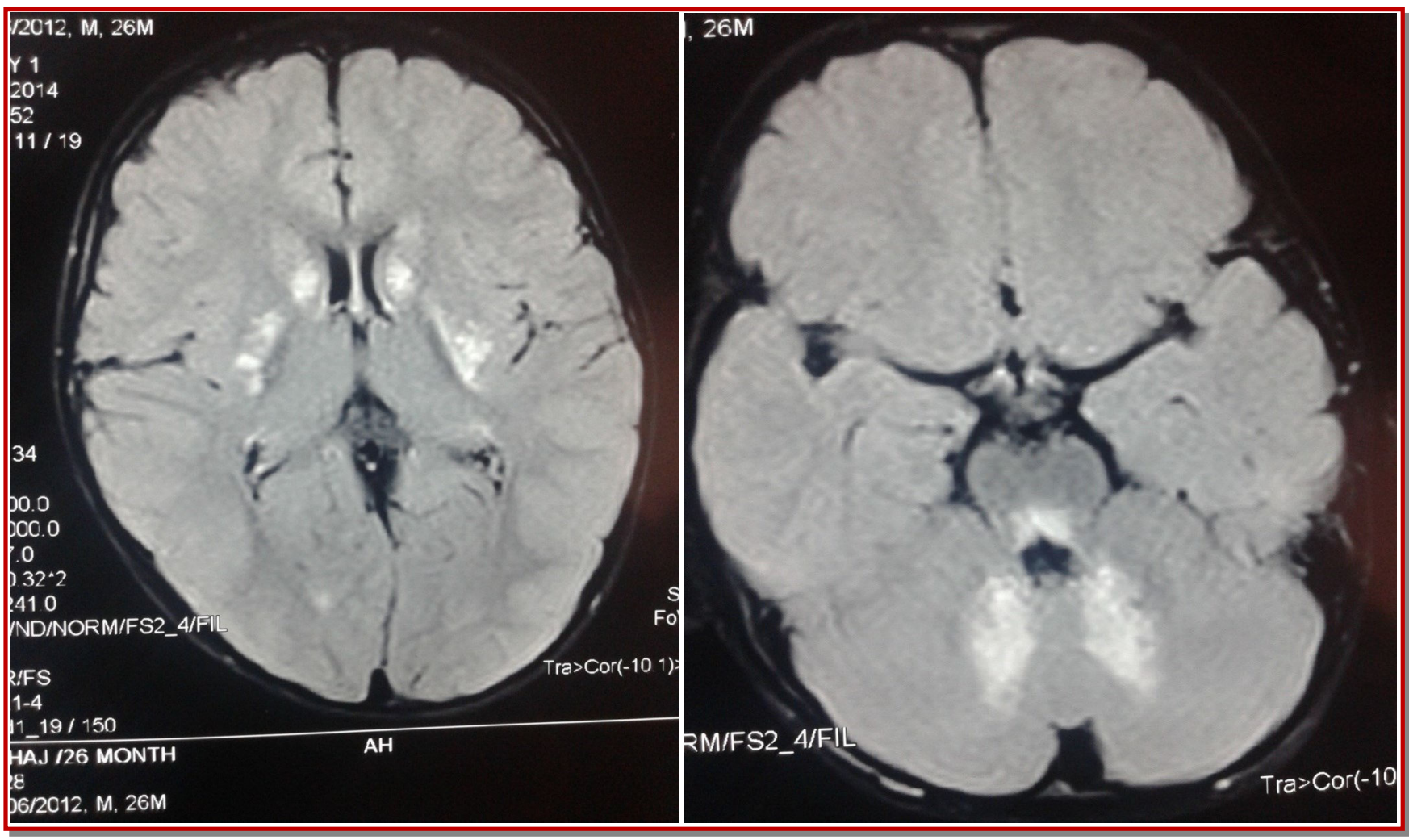

Figure 1: MR imaging of brain T1 with flair image revealed hyperintense lesion in both basal ganglia (left photograph), midbrain and dentate nuclei of cerebellum (right photograph)

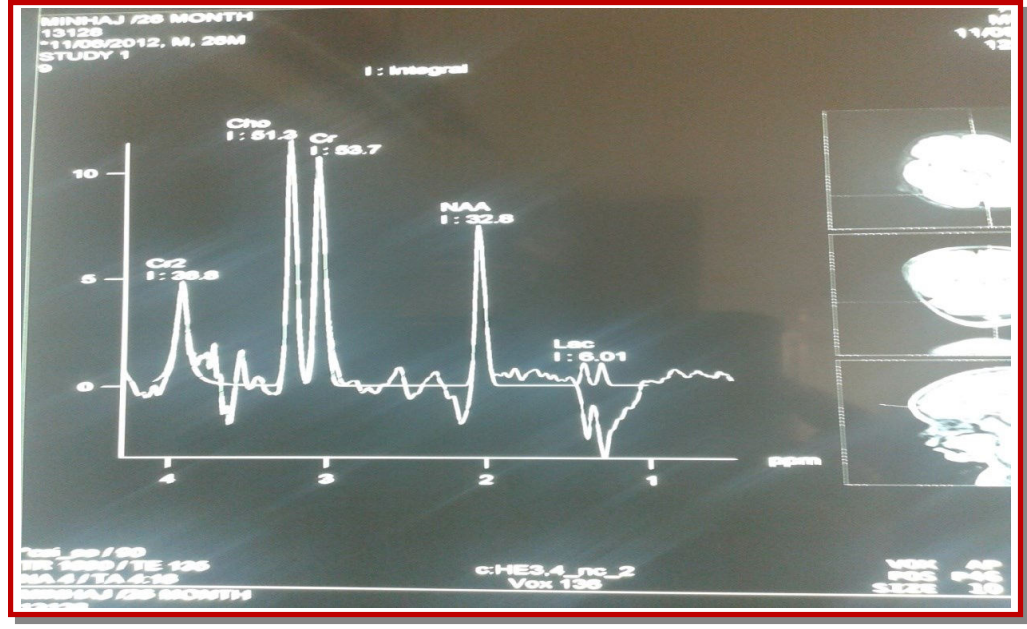

Figure 2: MR spectoscopy showing high lactate peak (double peak)

showed no specific findings.

\section{Treatment}

The patient was treated by cocktail therapy with coenzyme-Q, riboflavin, vitamin B complex, vitamin C, folinic acid, vitamin E and L-carnitine. Now he is on regular follow-up.

\section{Discussion}

Leigh's disease is a neurological disorder in chil- dren which is progressive in nature. The prevalence rate is 2.1 cases per $1,00,000.5$ The preschool incidence is 1 out of $32,000 . \underline{6}$ The age of onset of symptoms is usually $\leq 2$ years. It may be present in childhood but unusual in adult. It is presented with abnormal muscle tone, psychomotor regression, dystonia, weakness, ataxia, missed milestones or regression of the achieved milestones, visual loss, seizure and tachypnea.1,7,8 Children develop symptoms within the first year of life with difficulty in feeding, failure to thrive and vomiting.1

The defect in mitochondrial and nuclear DNA has been found that is similar clinical findings but occurs at different ages. $.7-10$ Clinical symptoms determine the area of the brain affected. The infantile form shows slowing in psychomotor development. Other symptoms are attention deficit, limb hypotonia, visual irregularity, nystagmus, unwellingness to eat, emesis and weight loss.7,8,10 Neurological symptoms are ataxia, apraxia, deficits of the pyramidal tract, myoclonus, ophthalmoplegia and respiratory difficulty. 7 Genetic irregularities are reported in $50 \%$ of all cases. There are many other biochemical irregularities which could lead to Leigh's disease.?

The search for mitochondrial DNA mutations and biochemical investigation of muscle biopsies are the gold standard for diagnosis.., 9

The most characteristic lesions found in the basal ganglia, involving especially the area of the caudate 
and putamen. Similar lesions are also seen in Wernicke's encephalopathy, multiple sclerosis, Wilson's disease, organic acidamia, mitochondrial encephalomyelopathy and various forms of intoxication. 11 In contrast to Wernicke's encephalopathy mamillary bodies are spared from pathological changes.11 A differentiation to Wilson's disease is possible through laboratory studies of the blood 8

In the basal ganglia, the putamen is particularly involved. In one series, $100 \%$ of the patients with proven subacute necrotizing encephalitis had putaminal involvement. 8 Two children with Leigh syndrome suspected clinically were reported and confirmed by MRI in 1996.12 Low attenuation in the putamina on CT is considered to be characteristic of the disease.11 In India, the role of CT scan of the brain in the diagnosis of Leigh syndrome was reported in 1991.11 In 2005, Hombal and Narvekar reported Leigh syndrome in a 3-year-old child with regression of milestones and involuntary movements. $\underline{13}$ The diagnosis is based on clinical presentation and neuroimaging with Leigh diseases.

No specific therapy for mitochondrial disorders in children is available. The aim of the treatment is to improve the production of ATP as well as to lower the lactate levels. The neurological status, in some patients, can be improved by administering thiamine.10 However, marked improvement has been reported following administration of riboflavin, that normalized the ATP production.11,13 The administration of coenzyme Q10, thiamine and Lcarnitine may cause a decrease in the severity of symptoms in a few cases. $\underline{11}$

Death usually occurs within a few years after onset of symptoms. It is due to progressive respiratory failure.., 10

\section{Conclusion}

In brain imaging, multiple brainstem nuclei/ associated with bilateral symmetric basal ganglia abnormalities in a child with neurological problems should prompt the clinician to consider Leigh syndrome and conduct investigations like estimation of lactate (blood, CSF) and respiratory chain enzymes.

\section{Ethical Issue}

Written and signed informed consent from the guardian was taken for publishing this case report.

\section{References}

1. Leigh D. Subacute necrotizing encephalomyelopathie in an infant. J Neurol Neurosurg Psychiatry. 1951; 14: 216-21.

2. Crimi M, Papadimitriou A, Galbiati S, Palamidou P, Fortunato F, Bordoni A, Papandreou U, Papadimitriou D, Hadjigeorgiou GM, Drogari E, Bresolin N, Comi GP. A new mitochondrial DNA mutation in ND3 gene causing severe Leigh syndrome with early lethality. Pediatr Res. 2004; 55: 842-46.

3. Cacic M, Wilichowski E, Mejaski-Bosnjak V, Fumić K, Lujić L, Marusić Della Marina B, Hanefeld F. Cytochrome c oxidase partial deficiency-associated leigh disease presenting as an extrapyramidal syndrome. J Child Neurol. 2001; 16: 616-19.

4. Menkes JH. Textbook of child neurology. 5th edi. London, Williams and Wilkins, 1995, p 850.

5. Rahman S, Blok RB, Dahl HH, Danks DM, Kirby DM, Chow CW, Christodoulou J, Thorburn DR. Leigh syndrome: Clinical features and biochemical and DNA abnormalities. Ann Neurol. 1996; 39: 343 -51 .

6. Darin N, Oldfors A, Moslemi AR, Holme E, Tulinius $M$. The incidence of mitochondrial encephalomyopathies in childhood: Clinical features and morphological, biochemical, and DNA abnormalities. Ann Neurol. 2001; 49: 377-83.

7. Huntsman RJ, Sinclair DB, Bhargava R, Chan A. Atypical presentations of Leigh syndrome: A case series and review. Pediatr Neurol. 2005; 32: 334-40.

8. Tomczak R, Rieber A, Zeitler H, Brambs HJ. Neugeborenesmit zerebraler symptomatic. Radiology 1996; 36: 591-92.

9. Savasta S, Comi GP, Perini MP, Lupi A, Strazzer S, Rognoni F, Rossoni R. Leigh disease: Clinical, neuroradiologic, and biochemical study of three new cases with cytochrome c oxidase deficiency. J Child Neurol. 2001; 16: 608-13.

10. Di Rocco M, Lamba LD, Minniti G, Caruso U, Naito E. Outcome of thiamine treatment in a child with Leigh disease due to thiamine-responsive pyruvate dehydrogenase deficiency. Eur J Paediatr Neurol. 2000; 4: 115-17.

11. Bar-Meir M, Elpeleg ON, Saada A. Effect of various agents on adenosine triphosphate synthesis in mitochondrial complex I deficiency. J Pediatr. 2001; 139: 868-70.

12. Spranger M, Schwab S, Wiebel M, Becker CM. Dasadulte Leigh-syndrom. Nervenarzt 1995; 66: 144-49.

13. Leung TF, Hui J, Yeung WL, Goh K. A Chinese girl with Leigh syndrome: Effect of botulinum toxin on dystonia. J Paediatr Child Health. 1998; 34: 480-82. 